\title{
Implementation of evidence-based stroke care: enablers, barriers, and the role of facilitators
}

\author{
This article was published in the following Dove Press journal: \\ Journal of Multidisciplinary Healthcare \\ 15 September 2014 \\ Number of times this article has been viewed
}

\section{Tara Purvis ${ }^{1,2}$ \\ Karen Moss ${ }^{2}$ \\ Sonia Denisenko ${ }^{3}$ \\ Chris Bladin 2,5 \\ Dominique A Cadilhac 1,2,4}

'Translational Public Health Unit, Stroke and Ageing Research Centre, Department of Medicine, Monash Medical Centre, School of Clinical Sciences at Monash Health, Monash University, Clayton, VIC, Australia; ${ }^{2}$ Stroke Division, Florey Institute of Neuroscience and Mental Health, Melbourne Brain Centre, Heidelberg, VIC, Australia; ${ }^{3}$ Commission for Hospital Improvement, Department of Health Victoria, VIC, Australia; ${ }^{4}$ Department of Medicine, University of Melbourne, Parkville, VIC, Australia; ${ }^{5}$ Eastern Health Clinical School, Monash University, Box Hill, VIC, Australia
Correspondence: Dominique A Cadilhac Level I/43-5I Kanooka Grove Clayton, VIC 3168, Australia

Tel +6I 395947583

Fax +6I 395946258

Email dominique.cadilhac@monash.edu

\begin{abstract}
A stroke care strategy was developed in 2007 to improve stroke services in Victoria, Australia. Eight stroke network facilitators (SNFs) were appointed in selected hospitals to enable the establishment of stroke units, develop thrombolysis services, and implement protocols. We aimed to explain the main issues being faced by clinicians in providing evidence-based stroke care, and to determine if the appointment of an SNF was perceived as an acceptable strategy to improve stroke care. Face-to-face semistructured interviews were used in a qualitative research design. Interview transcripts were verified by respondents prior to coding. Two researchers conducted thematic analysis of major themes and subthemes. Overall, 84 hospital staff participated in 33 interviews during 2008. The common factors found to impact on stroke care included staff and equipment availability, location of care, inconsistent use of clinical pathways, and professional beliefs. Other barriers included limited access to specialist clinicians and workload demands. The establishment of dedicated stroke units was considered essential to improve the quality of care. The SNF role was valued for identifying gaps in care and providing capacity to change clinical processes. This is the first large, qualitative multicenter study to describe issues associated with delivering high-quality stroke care and the potential benefits of SNFs to facilitate these improvements.
\end{abstract}

Keywords: stroke, stroke management, facilitators, qualitative, clinical practice, implementation

\section{Introduction}

Stroke is recognized as the leading cause of long-term adult disability ${ }^{1,2}$ and a primary cause of death. ${ }^{2}$ The burden of stroke in a community may be reduced through prevention strategies and a number of effective treatment interventions. Currently, the most successful and universally applicable intervention for acute stroke is organized management in the stroke units (SUs). ${ }^{3-6}$ Some of the reported reasons why SU care is more effective than general medical care for improving patient outcomes are associated with providing an environment that supports a greater adherence to a range of evidence-based interventions via a dedicated and specialized interdisciplinary clinical team..$^{3,47,8}$ However, in Australia, just over one-half of all patients with stroke receive care in a SU; ${ }^{9}$ access to other evidence-based treatments is variable. ${ }^{9}$ This is especially so for smaller hospitals, where patient admissions for stroke are infrequent, and where resources and specialist care are limited - especially in rural settings. ${ }^{10}$ This situation is similar to that of other countries. Determining the reasons and potential solutions for why it has been difficult to provide evidence-based care for stroke is not welldescribed in the literature. One approach may be to provide a dedicated position for coordination or facilitating uptake of evidence-based care. ${ }^{11}$ 
In Australia, the funding of acute public hospital care is the responsibility of the state government. Each state government has supported different initiatives to improve stroke care. In Victoria, a stroke expert advisory group, including government representatives and clinicians, was established, and a Stroke Care Strategy for Victoria was published in 2007. ${ }^{12}$ The Victorian Stroke Clinical Network (VSCN) was established in 2007 and made responsible for advancing the recommendations within the strategy, which included the need to provide stroke care in SUs, access to stroke thrombolysis, and use of protocols that conform to clinical practice guidelines. It was also recommended that clinical facilitators be employed for a time-limited period in selected hospitals where capacity to improve clinical care was considered a priority. From May 2008, eight facilitators, referred to as stroke network facilitators (SNFs), were employed at seven Victorian hospitals, for an initial period of 2 years.

The concept of using facilitators to implement clinical practice change has previously been reported. ${ }^{13,14}$ The promoting action on research implementation in the health services framework model ${ }^{13}$ focuses on three elements considered essential in the uptake of evidence-based practice in health services: evidence; context, which includes appropriate monitoring and feedback and the importance of leaders, among other aspects; and facilitation, where an individual, with the appropriate knowledge and skill, supports practitioners to change their practice.

Various types of clinical leadership roles exist for stroke care and are used interchangeably in the literature. We summarize these as follows for the purpose of this article: medical leadership that represents the main clinical opinion leader for medical care and is usually the stroke service team leader (or unit director) within a hospital; stroke care coordinators (SCCs), who may be nursing or allied health staff with senior specialist expertise, and are involved in providing education; and establishing and implementing services/protocols to ensure standardized and evidence-based care. Other terms used for this type of role include (but are not limited to) stroke clinical coordinator, a stroke liaison nurse, stroke nurse specialist, or clinical nurse consultant. The SCC clinical leadership role may or may not include a clinical patient load. ${ }^{15}$ The other commonly referred to clinical leadership role is that of the facilitator, a professional who is usually from a nursing or allied health discipline, and who provides direction, education, and support to staff within a stroke service to improve clinical care, ${ }^{14}$ but who does not take responsibility for care delivery.

Using clinical leadership roles to implement improvements in the management of various diseases at single sites, ${ }^{16,17}$ and within stroke care,${ }^{15}$ has been described. In these studies, Mellor et a ${ }^{17}$ and Larsen et $\mathrm{a}^{16}$ used existing nursing staff employed at the hospital and endorsed them in clinical leadership roles to implement changes in practice, which was successful at each site. However, the effectiveness remains unqualified on a multicenter scale. In the study conducted in New South Wales, Australia, similar SCC positions held by nurses or allied health professionals were provided as a permanent resource to the hospitals for improving the coordination of stroke care. ${ }^{15}$ However, it remains unclear if using a time-limited facilitator with nonclinical responsibilities is perceived as a worthwhile initiative, and whether it is effective for changing clinical practice for stroke across multiple sites. This was especially important since this concept for improving stroke care had not been used in Australia before, and these SNFs were not directly employed by the hospitals and may have been considered outsiders.

Identifying potential barriers and enablers is essential prior to implementing any strategy to improve care practices. Several barriers have been reported in stroke care, particularly hindering the implementation of stroke guidelines recommendations. Having limited resources - including lack of equipment, time, specialist and dedicated staff, ${ }^{18-20}$ and access to a stroke unit ${ }^{18}$ - is one of the most commonly reported barriers. Other barriers include: limited knowledge and skill; professional beliefs about capabilities; ${ }^{21}$ and the ability to interpret research findings. ${ }^{22}$ Although there is existing knowledge in this area, there is limited research that has been published examining barriers across metropolitan and rural settings and in both the acute and subacute sectors.

The VSCN commissioned this research, led by Cadilhac et al, with the aims of describing the clinical practices for stroke across the continuum of care, at a range of hospitals with limited organizational resources, and to determine the local enablers and barriers to providing evidence-based stroke care. We also sought to determine, within the first 6 months of SNF placements, whether the role was acceptable to hospital staff and if there had been any early achievements. These data would assist in providing evidence for the ongoing implementation strategy and also in providing a baseline assessment of clinician beliefs and attitudes for future follow-up studies.

\section{Methods}

We used semistructured interviews with clinicians as part of a qualitative research design. This methodology enhances the ability to describe fully why care practices may or may not happen through the enrichment of knowledge by the firsthand accounts of those working in the field. ${ }^{23}$ 


\section{Selection of hospitals and organization of care}

In Australia, similar to other countries, stroke care is often provided in different phases. Generally, patients are initially treated in the emergency department (ED) and then admitted to an acute stroke unit (or medical ward, if the hospital does not have a stroke unit or neurology ward), for $\sim 7-10$ days. If inpatient rehabilitation is required, this is usually provided in a separate subacute setting, such as a rehabilitation ward or hospital. Rehabilitation may also be offered in a community setting, such as in home, outpatient, or day center, usually for up to 4 weeks. Hospitals that offer different phases of care may be formally administered by the same health board or executives (as part of a network of health services); therefore, it is possible to assess and influence all phases of care.

The seven hospitals selected to have an SNF appointed to their network of health services - with the objective of improving stroke care from the emergency department through to the subacute phases - were found to have a large stroke patient volume and limited physical and organizational infrastructure for stroke. Among these seven hospitals, three were in the metropolitan area, with population centers of $>100,000$, and four were in regional locations, which were in larger rural centers with a population of $>25,000$ but $<100,000 .{ }^{24}$

\section{Background of SNFs}

The SNFs placed in these health services had clinical backgrounds in nursing or allied health disciplines. Their role was to develop and to oversee the operation of protocols and training to support the implementation of the evidence-based recommendations from the Stroke Care Strategy for Victoria at the selected hospitals. Local evidence derived from a gap analysis performed by each SNF identified areas for prioritizing improvements in the provision of evidence-based stroke care and was used in monitoring-feedback cycles at each hospital.

\section{Interview process}

Purposive sampling was used whereby the staff who had direct involvement with providing care to patients with stroke or who were senior executives with decision-making authority to influence processes of care from each hospital were identified and invited to participate in the interviews. The SNF was best placed to know who the most-appropriate hospital staff would be and so approached the interviewees directly following instruction by the researchers regarding the scope of the respondents needed. Participation in the interviews was voluntary. The same researcher (DC) facilitated all the interviews. The researchers (DC, KM) visited each hospital on a mutually agreed date to conduct the prearranged faceto-face staff interviews that lasted from 45-60 minutes. The interviews were conducted from October-November 2008.

Most of the interviews were performed as a focus group or, occasionally, on an individual basis, depending on the staff availability or preference. The respondents included a diverse group of clinical staff directly involved in caring for patients with stroke. It was important to capture the whole continuum of care from the ED, to acute care, and then to subacute care, which involved rehabilitation services. Because executive support is important for changing policy and practice, ${ }^{18,25}$ executive staff were interviewed where available. Each focus group generally comprised staff working within a particular area or discipline, for example, EDs, acute wards, rehabilitation, health directors, or allied health professionals.

\section{Data collection}

The interview schedule comprised 15 primary open-ended questions within eight sections covering the following topics: feedback on the SNFs role including the perceived benefits of the position and areas of the role that could be improved; care practices for stroke; patient transfers and organizational models of care; strengths and barriers to the implementation of evidence-based stroke care; elicitation of the most pressing needs for the health service; the ability to monitor progress; and formal and informal linkages between service providers within and between hospitals at the time of the interviews. In addition, the composition of the stroke workforce and other available resources at each hospital were obtained.

The interview schedule used was not specifically underpinned by a single theoretical framework, since its initial development ${ }^{26}$ predated much of the work that has evolved in the field of implementation science. The questions proposed were adapted from our earlier work in the late 1990s and were subsequently assessed and adjusted with input from the VSCN managers and the SNF expert working party, which ensured all the important concepts specific to this project were captured. When we assessed the interview schedule used in our study against the checklist recently published by Flottorp et al, ${ }^{27}$ our questions were found to cover each of the seven recommended domains including: guideline factors; professional interactions; resources; individual health professional factors; patient factors; social factors; and organizational characteristics. ${ }^{27}$

The same interview schedule was used in all interviews. Because the interview schedule was comprehensive to cover 
the entire scope of stroke care, not all questions were relevant to each focus group. Depending on the type of participants in each focus group, only the questions of most relevance were asked. Respondents were informed that their responses would be pooled and deidentified.

During the interviews, data were directly recorded on the paper-based interview schedule by at least two people. The interview schedule was designed to facilitate note taking to ensure all responses would be captured within the time available for each interview. The interviewer (DC), who is an experienced clinical researcher in the field of stroke, was able to knowledgeably probe the interviewees for information to ensure the research questions were adequately covered. The second note taker was a research assistant (KM) who provided the ability to verify that the notes taken by the interviewer included all the key issues being reported. Data from each set of notes were then transcribed into a separate Excel (2003 version; Microsoft Corporation, Redmond, WA, USA) worksheet for each interview. The transcripts were returned to the respondents via a nominated focus group representative. Respondents could then add any further information to the transcript or change anything if they felt it was not an accurate representation of the information they had intended to convey. This method of verification provided the respondents with the opportunity to correct any potential misreporting of responses, thereby assisting with the reliability of the data. ${ }^{23}$

The SNFs also completed a supplemental questionnaire. This was designed to elicit information from the SNF's point of view, as well as to provide information on objective organizational characteristics of the hospital, for example, the number of beds. These responses were incorporated into the final dataset for each hospital.

\section{Data analysis}

Each hospital was given a unique identification number, and a master file combining the data from each hospital was created. An inductive approach to the thematic analysis was undertaken; whereby, one researcher performed a line-by-line analysis of the text to identify the main themes and subthemes, and this was used to develop a coding tree. The data were then coded using the coding tree. ${ }^{28}$ A second researcher also coded the transcripts, and then the results of the coding were cross-checked. Any discrepancies were discussed and resolved. Figure 1 shows the process of analysis and reporting of the qualitative data.

The Victorian Department of Health determined that this study did not require a formal ethics committee review, since the purpose was related to quality assurance, no patient data were being used, no tape recordings were made of the interviews, and all data were to be analyzed in an aggregated and deidentified manner. ${ }^{29}$ Instead, it was a requirement that an appropriate executive representative provide approval for the interviews to be conducted at each hospital. These approvals were obtained prior to any interviews being conducted.

\section{Results}

The findings from this study are presented below with some respondent quotes used to enhance the interpretation of the major themes and subthemes identified. When referring to data from a specific hospital $(\mathrm{H})$, this has been denoted in the text by $(\mathrm{H} 1),(\mathrm{H} 2)$, etc.

\section{Characteristics of the respondents}

Eighty-four health professionals participated in 33 focus group interviews across the seven hospitals. Overall, the median number of respondents per focus group was two with an interquartile range of two (minimum, one; maximum, eight). Additionally, six SNFs completed supplemental questionnaires. The average number of years the respondents worked in their current position was 4.4 years (minimum, 2 months; maximum, 300 months). The majority of respondents represented the acute setting $(32 \%)$ or the subacute setting (31\%), while 13\% worked across the continuum of care in both the acute and subacute settings (Figure 2). In total, $45 \%$ were allied health, 16\% were nursing staff, $27 \%$ were department or unit managers/executives, and $12 \%$ were physicians or medical staff.

\section{The SNF, clinical practices, and perceived enablers and barriers to providing optimal stroke care}

The major themes that emerged from the interviews relating to clinical practices included the stroke services across the continuum of care, use of other evidence-based practices, and staff resources, as well as the SNF role (Table 1). The following provides an overview of the themes and subthemes that emerged for these topics. Table 1 presents a summary of the perceived barriers and enablers to providing evidencebased stroke care.

\section{Stroke services}

At the time of the interviews, only two of the seven hospitals had an established formally recognized acute SU. However, it was reported that limited stroke beds within the SU was affecting access, so not all patients were being admitted to the 


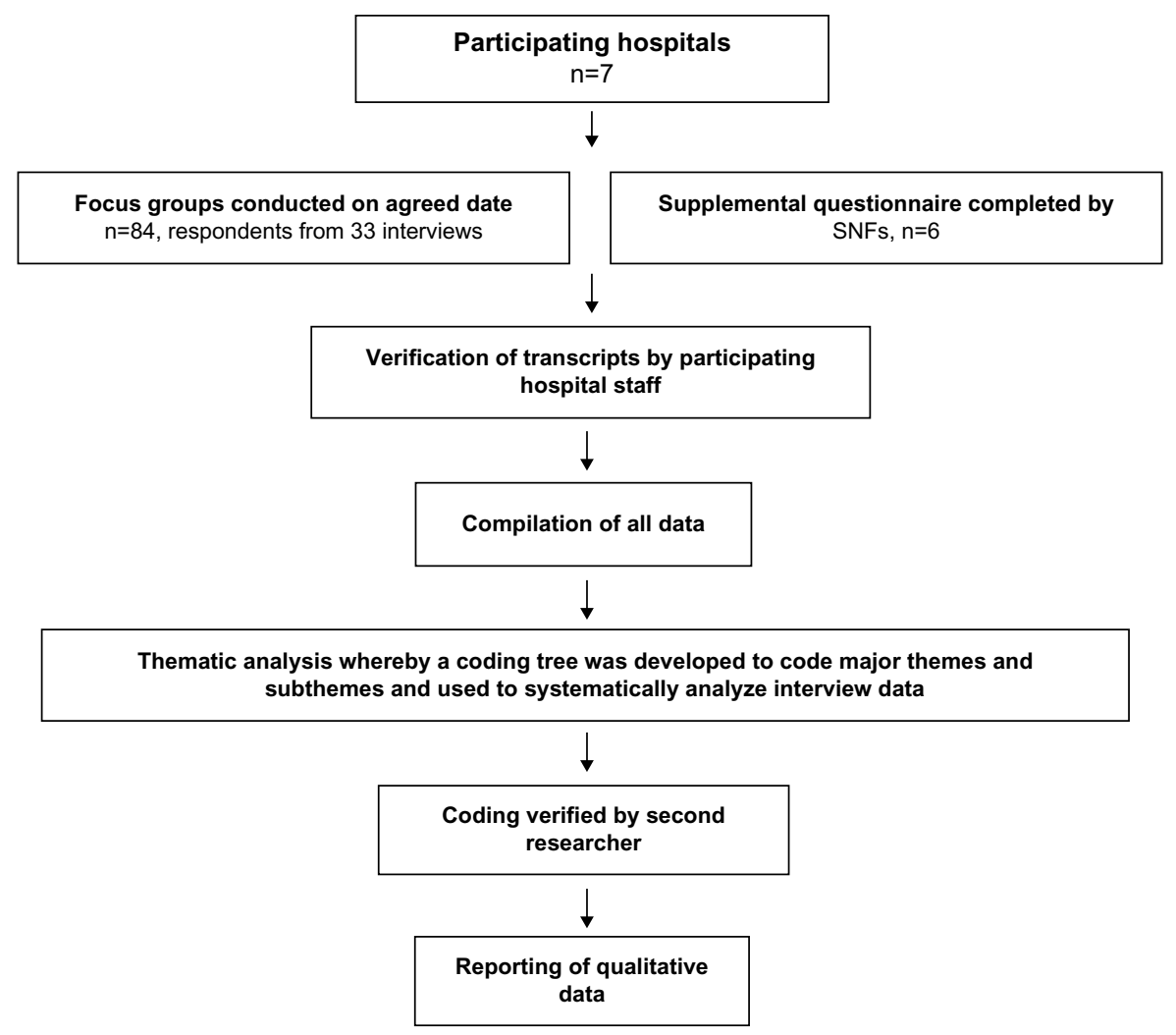

Figure I Process of analysis of the qualitative data.

Note: Illustrates the process and analysis used for the qualitative data collected.

Abbreviation: SNFs, stroke network facilitators.

SU at these hospitals. There was mixed access to dedicated beds for stroke within general or neurology wards at the remaining five hospitals (Table 2). Staff felt that not having an SU was a barrier to providing evidence-based care, and it was the view of some respondents that an SU would enhance the profile of stroke at their health service, which, in turn, would assist in attracting neurology-trained specialist staff.

Patients from each hospital had access to a range of inpatient subacute rehabilitation services, none of which had dedicated beds for stroke. A variety of outpatient services were

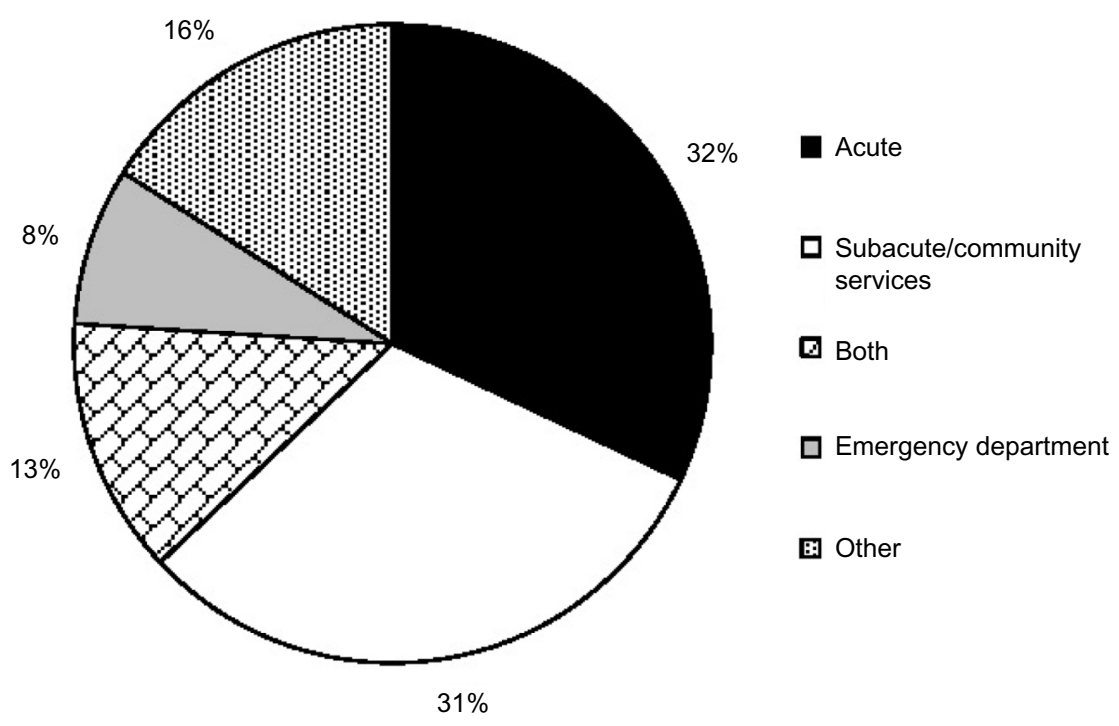

Figure 2 Respondent work settings.

Notes: "Other" includes heads of department or executives. "Both" includes those who work in the acute and subacute settings. 
Table I Main themes, subthemes to emerge from all focus groups interviews and common perceptions about strengths, limitations of stroke care

\begin{tabular}{|c|c|c|c|}
\hline Theme & Subtheme & $\begin{array}{l}\text { Perceived enablers to providing } \\
\text { evidence-based stroke care }\end{array}$ & $\begin{array}{l}\text { Perceived barriers to providing evidence-based } \\
\text { stroke care }\end{array}$ \\
\hline \multirow[t]{2}{*}{$\begin{array}{l}\text { Stroke } \\
\text { services }\end{array}$} & Acute services & $\begin{array}{l}\text { Stroke unit providing focus for acute stroke care and } \\
\text { team-based approach across all stages of care. Having } \\
\text { executive support to enhance stroke service model. }\end{array}$ & $\begin{array}{l}\text { Lack of dedicated stroke unit. Limited number of stroke } \\
\text { unit beds can affect access. }\end{array}$ \\
\hline & $\begin{array}{l}\text { Rehabilitation } \\
\text { services }\end{array}$ & $\begin{array}{l}\text { Community rehabilitation program for continuing } \\
\text { interventions postdischarge. Good structures, } \\
\text { documentation, and framework of rehabilitation. }\end{array}$ & $\begin{array}{l}\text { Lack of resources including equipment and outpatient } \\
\text { services for rehabilitation. No systems of care for } \\
\text { stroke across the continuum of care. }\end{array}$ \\
\hline \multirow[t]{4}{*}{$\begin{array}{l}\text { Use of other } \\
\text { evidence-based } \\
\text { practices }^{\mathrm{a}}\end{array}$} & $\begin{array}{l}\text { Thrombolysis } \\
\text { service }\end{array}$ & $\begin{array}{l}\text { Community awareness surrounding thrombolysis } \\
\text { to avoid delays in patient presentation to hospital. } \\
\text { Ability to safely and effectively deliver } \\
\text { intravenous thrombolysis } 24 / 7 \text {. }\end{array}$ & $\begin{array}{l}\text { For sites which have a thrombolysis service, the inability } \\
\text { to administer intravenous thrombolysis, or inconsistent } \\
\text { administration. Shortage of neurologists and resistance } \\
\text { from ED doctors. Lack of formalized guidelines or } \\
\text { protocols for the administration of thrombolysis. }\end{array}$ \\
\hline & Care pathways & $\begin{array}{l}\text { Protocols and guidelines in stroke care to streamline } \\
\text { management. Improved awareness of stroke } \\
\text { protocols and strategies across the hospital. }\end{array}$ & $\begin{array}{l}\text { Need for constant updating and having a staff member } \\
\text { with the capacity to do this. Inconsistent use of care } \\
\text { pathways. } \\
\text { Generic pathways not as effective as stroke specific. }\end{array}$ \\
\hline & $\begin{array}{l}\text { Education } \\
\text { for patients } \\
\text { and family }\end{array}$ & $\begin{array}{l}\text { More comprehensive coverage in subacute } \\
\text { setting. Education consistently provided } \\
\text { by stroke nurse. }\end{array}$ & $\begin{array}{l}\text { Lack of formalized process led to inconsistent provision } \\
\text { of education. Lack of time for staff to provide education } \\
\text { in acute setting. }\end{array}$ \\
\hline & $\begin{array}{l}\text { Professional } \\
\text { development } \\
\text { for staff }\end{array}$ & $\begin{array}{l}\text { Access to ongoing education and entitlements } \\
\text { for professional development leave or grants. }\end{array}$ & $\begin{array}{l}\text { Lack of funding for professional development } \\
\text { opportunities for allied health and nursing. }\end{array}$ \\
\hline \multirow[t]{2}{*}{$\begin{array}{l}\text { Staff resources } \\
\text { and attributes }\end{array}$} & $\begin{array}{l}\text { Access to } \\
\text { specialist clinical } \\
\text { staff and clinical } \\
\text { leaders }\end{array}$ & $\begin{array}{l}\text { Strong medical support improves likelihood } \\
\text { of thrombolysis administration or use of stroke } \\
\text { pathway. Commitment by staff and a great } \\
\text { "teamwork culture" among all disciplines. } \\
\text { A stable team. }\end{array}$ & $\begin{array}{l}\text { Lack of strong medical leadership or absence of a } \\
\text { neurologist to support the stroke service - leads to } \\
\text { delays in clinical decision making. Frequently rotating } \\
\text { staff mean more time and resources required to up skill } \\
\text { and provide consistent patient care. Limited access to } \\
\text { allied health on weekends. }\end{array}$ \\
\hline & $\begin{array}{l}\text { Workforce } \\
\text { demands }\end{array}$ & $\begin{array}{l}\text { Specialist nurses and champions for stroke who } \\
\text { can service as a clinical leader and assist the SNF. }\end{array}$ & $\begin{array}{l}\text { Inadequate access to allied health staff, with no } \\
\text { replacement for allied health staff when on leave. }\end{array}$ \\
\hline \multirow[t]{4}{*}{ SNF role } & $\begin{array}{l}\text { Scope of } \\
\text { responsibilities }\end{array}$ & $\begin{array}{l}\text { Valuable in identifying gaps in current service that } \\
\text { provide focus for improvement efforts. Even in first } \\
6 \text { months of SNF tenure, enhancements to stroke care } \\
\text { are evident. Assisted in engaging all key stakeholders, } \\
\text { required to make changes within hospitals. }\end{array}$ & $\begin{array}{l}\text { Role could be enhanced if additional clinical support } \\
\text { was available, eg, to assist with staff education. }\end{array}$ \\
\hline & $\begin{array}{l}\text { Executive } \\
\text { support }\end{array}$ & $\begin{array}{l}\text { Ongoing commitment to the role within the } \\
\text { hospital and provided support for SNF initiatives. }\end{array}$ & $\begin{array}{l}\text { Restricts ability to make changes to policy and practice } \\
\text { or make resources available to support improvement } \\
\text { efforts. }\end{array}$ \\
\hline & $\begin{array}{l}\text { Part-time } \\
\text { employment }\end{array}$ & $\begin{array}{l}\text { Allowed time to facilitate and streamline processes } \\
\text { to improve care practices. Not bogged down with } \\
\text { clinical workload responsibilities. }\end{array}$ & $\begin{array}{l}\text { Influenced the capacity to meet all the objectives of the } \\
\text { role as time limited. Inadequate cover for such large } \\
\text { regions with limited time allocation. }\end{array}$ \\
\hline & $\begin{array}{l}\text { Length of } \\
\text { appointment }\end{array}$ & & Insufficient time period to achieve all objectives. \\
\hline
\end{tabular}

Note: an addition to care in a stroke unit.

Abbreviations: SNF, stroke network facilitator; ED, emergency department.

available, including three hospitals that offered a transitional care program to coordinate health care for patients over the longer term (Table 2). The main issues for both the acute and subacute settings were: out-of-hours bed management decisions which affected patient flow throughout the service; a lack of clear and consistent ward leadership; financial constraints which limited staff resources and access to equipment for rehabilitation; and the frequently rotating staff (eg, every
3 months), which made the time commitment required to up skill staff detrimental to making improvements in stroke services. These factors made it difficult to provide consistent and high-quality stroke care.

\section{Use of other evidence-based practices}

The subthemes of thrombolysis services, use of standardized care pathways and protocols, education for patients 
Table 2 Characteristics of stroke care within each hospital

\begin{tabular}{|c|c|c|c|c|c|c|c|}
\hline Hospital & Location & $\begin{array}{l}\text { Stroke } \\
\text { unit }\end{array}$ & $\begin{array}{l}\text { Beds allocated } \\
\text { for stroke }\end{array}$ & Thrombolysis & $\begin{array}{l}\text { MDT } \\
\text { meetings }\end{array}$ & $\begin{array}{l}\text { Acute stroke } \\
\text { care pathways }\end{array}$ & $\begin{array}{l}\text { Access to } \\
\text { rehabilitation }\end{array}$ \\
\hline $\mathrm{I}$ & Metropolitan & $\begin{array}{l}\text { Mixed } \\
\text { views }\end{array}$ & $\begin{array}{l}\text { None, but capacity for } \\
\text { I5-20 patients with } \\
\text { stroke in neurology ward }\end{array}$ & Yes, infrequently & 4 times/week & Yes & $\begin{array}{l}\text { Offsite inpatient; } \\
\text { access to outpatients }\end{array}$ \\
\hline 2 & Metropolitan & No & Mixed views: $0-4$ beds & No & Weekly & Yes & $\begin{array}{l}\text { Offsite inpatient; } \\
\text { access to outpatients }\end{array}$ \\
\hline 3 & Regional & No & 4 beds & Yes, infrequently & Weekly & Yes & $\begin{array}{l}\text { Offsite inpatient; } \\
\text { access to outpatients }\end{array}$ \\
\hline 4 & Metropolitan & No & None & Yes & Daily & Yes & $\begin{array}{l}\text { Offsite inpatient; } \\
\text { access to outpatients }\end{array}$ \\
\hline 5 & Regional & No & None & No & Weekly & Yes & $\begin{array}{l}\text { Onsite inpatient; } \\
\text { access to outpatients }\end{array}$ \\
\hline 6 & Regional & Yes & 3 beds & Yes, infrequently & Weekly & Yes & $\begin{array}{l}\text { Onsite inpatient; } \\
\text { access to outpatients }\end{array}$ \\
\hline 7 & Regional & Yes & 4 beds & No & Weekly & Yes & $\begin{array}{l}\text { Offsite inpatient; } \\
\text { access to outpatients }\end{array}$ \\
\hline
\end{tabular}

Abbreviation: MDT, multidisciplinary team.

with stroke, and professional development for staff were identified when exploring questions about the ability to provide evidence-based practices.

\section{Thrombolysis services}

Respondents reported that intravenous thrombolysis had been administered infrequently at four of the hospitals, or not at all (Table 2). There was an overall perception among respondents that the use of thrombolysis was hindered by a shortage of neurologists and "... resistance and lack of intent" from some ED doctors who do not support the use of thrombolysis. This was compounded by a lack of formalized guidelines or protocols and the absence of an acute SU. The utilization of thrombolysis as a potentially effective treatment for acute stroke was also limited by a lack of community, general practitioner, and ambulance staff education and awareness of the time constraints associated with thrombolysis treatment. This created potential delays in ensuring patients arrived within the critical time window (ie, 4.5 hours of stroke onset). In regional hospitals, time was the major perceived limitation in the administration of thrombolysis. This was associated with patient travel time to get to the hospital, as well as the time required to access limited neurology and radiography services, especially during out-of-hours when "the travelling is at least an hour into regional areas, and no-one lives nearby."

\section{Use of standardized care pathways or protocols}

Six hospitals had initiated standardized stroke care pathways in the ED. However, many staff reported that these required updating, with frequent accounts of inconsistent use. Where an official stroke pathway was not in place in the ED, it was perceived that some patients had longer stays in the ED waiting for diagnostic tests. All hospitals used care pathways or protocols for stroke management in the acute wards. In the subacute settings, pathways and protocols were usually generic, rather than stroke-specific. None of the care pathways was used across the different stages of inpatient care. The lack of formalized protocols for transferring patients between hospitals across a region was also reported. It was identified that greater efficiencies and timeliness of care could be achieved by having formalized transfer protocols.

\section{Education for patients with stroke and carers/families}

In the acute setting, there was an overall perception that education for patients with stroke and their carers/families was available on an informal basis, described as ad hoc, and was generally "... inconsistent and not evidence-based" $(\mathrm{H} 1, \mathrm{H} 2, \mathrm{H} 3, \mathrm{H} 4, \mathrm{H} 7)$ and often poorly documented (H3, H6). Time limitations were perceived to prevent staff providing education. However, it was stated that allied health staff would often provide discipline-specific education during therapy sessions. It was generally believed that there was more structured and wider education available for patients and their carers/family members in the subacute setting than in the acute setting (H2, H4, H5, H7); “... education is all covered there (in subacute settings) and in more detail."

\section{Professional development opportunities for staff}

Respondents at each hospital described access to ongoing educational opportunities and entitlements for professional development leave as important for advancing 
clinical practice. This included the ability to participate in conferences, workshops, seminars, stroke-specific education days, and internal and external stroke education forums. However, financial support to attend external educational opportunities was variable, depending on discipline and/or hospital funds available for such activities. Only one hospital in this study did not raise lack of funding as an issue for participating in professional development (H6).

Nurses appeared to be the most disadvantaged clinical group with respect to having external professional development opportunities funded by their hospital; “... there is no nursing budget to send staff to external courses or conferences ... only 30 minutes is allocated for internal education (for nurses)" (H2). Similarly, respondents from H7 stated there is "... no budget for external education programs for nursing staff ... funds have to come from the ward funds."

\section{Staff resources}

The main subthemes relating to staff resources included access to specialist clinical staff and clinical leaders and workload demands.

Respondents reported that there was limited access to neurologists for patients with stroke at their hospital, and there was variable interest in stroke by doctors responsible for treating patients with stroke. This was mainly due to the heavy workloads, and therefore, limited capacity to focus on stroke. This created a perceived lack of medical leadership for stroke, and inconsistent patient care; “... the lack of one leader for stroke limited continuity of care." Respondents from subacute settings felt that having rehabilitation specialists with a particular interest in stroke would improve care in stroke rehabilitation. "Some (patients) end up in rehabilitation making little progress ... there is no one person to make decisions ... a stroke (rehabilitation) specialist would formalise decisions for patients."

Dedicated nursing or allied health positions for stroke were also lacking. There was an overwhelming sentiment from respondents that a dedicated stroke nurse specialist would enhance patient management and achieve sustainable changes in practice. It was perceived that stroke specialist nurses could provide clinical leadership for stroke and ensure that evidence-based practice is delivered and monitored. Staff also suggested that specialist nurses could assist with the education of both staff and patients/families in the acute and the subacute settings, and improve organization, flow, and coordination of care and discharge planning. At the time of the interviews, only one of the hospitals (H6) had a nurse specialist for stroke. Staff from this hospital reported that " ${ }^{\text {... }}$ we couldn't have achieved and maintained our improvements since 2003 without the dedicated stroke nurse," and they were described as being "... extremely important in the provision of evidence-based stroke care."

Shortages of dedicated allied health staff were an issue consistently raised. It was felt there were not enough staff resources to cover the patient load: "Each team sees many other patients besides stroke, and other caseloads are often prioritised over neuro[logical] conditions."

In addition, there was often no backfill for allied health when staff took leave, which was perceived to "... impact on the levels of input and quality (of therapy)." All hospitals reportedly had variable access to physiotherapy, speech pathology, occupational therapy, social work, therapy assistants, and dietetics. Demands on physiotherapy services were stated to be "... heavy," and social work positions were described as “... stretched.” Overall, there was limited access to psychology and neuropsychology clinicians, which added to the demands on social workers, who were often expected to fulfill aspects of the psychologist's roles. Respondents at most hospitals reported very limited or no access to allied health on weekends. Not having speech pathologists to perform comprehensive dysphagia (swallowing) assessments during weekends was a great concern, because it could result in patients being left for long periods of time unable to eat or drink. Respondents stated that variable and untimely access to allied health staff could lead to delays in instigating the initial rehabilitation program, and that the patient potentially "... could go backwards."

\section{The SNF role}

The main subthemes to emerge from the discussion on the SNF role included their scope of responsibilities, whether they had executive support, their part-time employment status, and the length of their appointment.

At the time of the interviews, most of the SNFs had only been employed for about 6 months. The majority of respondents unanimously considered the SNF role as valuable for improving stroke care and that it had provided an enhanced focus for stroke care. The SNF had driven the formation of steering committees and had garnered executive support to prioritise stroke care initiatives. The SNF was considered a clinical leader who could: increase stroke awareness and education among staff; identify gaps in care across the hospital and broader health service network; facilitate change in clinical practice and processes (eg, through developing business plans); and provide performance data in relation to stroke care that was able to be contrasted to other hospitals. 
It was also perceived that the SNF could be a champion who could advocate for more resources and equipment. Overall, the SNF's role was thought to be "... to co-ordinate and to facilitate, to drive the process, but not to do the work." The SNF was perceived as "... someone to look across the continuum, someone to develop systems and to try to get initiatives happening," and "... an external person (from the hospital) who is not bogged down with caseload."

A perceived limitation of the SNF role was the concern that the 2-year, part-time position would be insufficient to achieve all the desired objectives. Where relevant, the geographical size of the region was also a concern; "... this is a huge role in a short time frame for a large regional area. Time is lost in travel in the community."

\section{Discussion}

This is the first large, qualitative multicenter study, across both acute and subacute settings with respondents from metropolitan- and regional-based hospitals, which provides detailed information on the barriers and enablers to providing evidence-based stroke care, and the initial effect of facilitators on service provision. Our findings may be of relevance to other countries or regions where the health system for stroke is similar to that provided in Victoria, Australia.

Very few studies have been conducted across a diverse range of hospitals on the perceived issues in delivering evidence-based stroke care. ${ }^{18,20,21}$ We have described from the perspective of clinicians the difficulties faced in providing evidence-based stroke care across the continuum of care in both metropolitan and regional hospitals. These barriers can be addressed in future strategies looking to implement changes in practice. We were also able to highlight the perceived enablers for improving stroke care in these hospitals. Further, little is known about the effectiveness of time-limited clinical leadership positions for implementing evidencebased stroke care. Different to other reported strategies using SCCs to improve stroke care, ${ }^{15}$ the clinical leadership role of the SNF, was time-limited and did not include direct patient management. For the first time, we describe initial feedback on this nonmedical clinical leadership position that was focused on improving stroke care across multiple sites.

Numerous factors, including a lack of resources, limited knowledge and skills, individual attitudes to providing specific interventions, having managerial or executive support, and the presence of an advocate or clinical leader have been reported to affect the implementation of stroke guidelines in various areas of stroke care. ${ }^{18,20,21}$ Even though some of these studies were conducted at a single site, ${ }^{21}$ or were focused on one aspect of care, ${ }^{19}$ our results are consistent with the majority of these findings. In particular, studies whose results identified the influence of limited resources ${ }^{18,20}$ and the need for clinical advocates and greater managerial support. ${ }^{19}$ However, as a point of difference, our results also provide new insights into the perceived influence of a facilitator position for improving stroke care. Including data from a diverse sample of clinicians working across acute and subacute phases of stroke care from multiple hospitals means our results may be relevant to hospitals in other countries where care may be fragmented or uncoordinated for stroke. ${ }^{30}$

We found that the lack of dedicated acute SUs at most of the hospitals was a major barrier. This is a situation that is also common in other countries where access to SUs is variable. In our study, it was felt that a formalized SU would concentrate the resources for stroke care and enable greater consistency of care to improve stroke outcomes. Another important factor identified was the inconsistent use of thrombolysis. Van Wijngaarden et $\mathrm{al}^{31}$ propose that the organizational culture impacts on the use of thrombolysis at individual hospitals. Similarly, our findings also suggest that a major impediment to the use of intravenous thrombolysis was related to organizational culture, in terms of the lack of clinical medical leadership and the differences of opinions among medical physicians. Consistent with our findings, van Wijngaarden et $\mathrm{al}^{31}$ proposed that the use of stroke pathways and protocols - supported by clinical leaders and regular monitoring and feedback - may increase the use of thrombolysis treatment in stroke. Interestingly, pathways and protocols had been used at most hospitals during our research; therefore, our data provide better information on what clinicians actually think about these strategies. One more critical factor we found for addressing barriers to providing evidence-based care for stroke was ensuring that organizational support at an executive level was available to achieve overall change.

In accordance with previous research, ${ }^{16,17,32,33}$ we provide evidence on the importance of clinical leadership roles for improving stroke care. Having dedicated medical leadership by a physician who has specialized in stroke is often referred to as important for ensuring evidence-based care is delivered. ${ }^{31,34}$ In the absence of a specialist medical physician being available to lead a stroke service, or having limited time to implement systemwide change, nonmedical clinical coordinators may add value or be a reasonable substitute for this role. ${ }^{15-17}$ In addition, dedicated stroke nurse specialists, while still having direct patient contact, have the knowledge and skills to facilitate and support improvements to interdisciplinary stroke care. 
In the current study, we found that where a stroke nurse specialist role had already existed in one hospital, respondents reported numerous benefits in aspects of care. Internationally, similar roles to the stroke nurse specialist include the stroke nurse navigator ${ }^{35}$ and clinical nurse leader ${ }^{36}$ positions. Research has shown that the development of specialist and advanced nursing roles have elevated levels of evidence-based care practice within stroke services ${ }^{37}$ and enhanced patient satisfaction with the continuity of stroke care from acute care to postdischarge care. ${ }^{38}$

While the SNF was considered to be providing clinical leadership, with the time-limited nature of the position, many respondents acknowledged the need for other clinical staff to step into this leadership role to achieve sustainable change in practice. The primary responsibility of the SNF was to develop and oversee the implementation of protocols to support the recommendations of the Stroke Care Strategy for Victoria.

Developing recommendations or guidelines alone is not effective in changing clinician behavior or hospital processes ${ }^{39}$ The challenge is to systematically ensure these guideline recommendations are translated into clinical practice and - through a system of monitoring and review - are sustained. Although the SNFs had only been in post for up to 6 months at the time of the interviews, significant gains had been reported. The SNFs had played an integral role in establishing and improving stroke unit care at the health services, thus facilitating the translation of policy into evidence-based practice. The gap analyses had been used to identify service strengths and gaps and as mechanisms to highlight directions of change. Steering committees and working groups were formed to prioritize areas for improvement over the SNF tenure. However, respondents felt there was a need to increase the current working hours of the SNF, as well as the duration of the role if all the service enhancements were to be achieved and, importantly, sustained. As a result of this study, the SNFs received additional funding to extend their tenure to 3 years. A further evaluation of these seven hospitals would be useful to examine whether the changes that occurred at these hospitals while the SNFs were employed were sustained after the SNF tenures finished.

This qualitative research used purposeful sampling. A large number of interviews and volume of data were obtained. Saturation of issues relating to each aim was achieved. Nonetheless, it is possible that some issues may have been missed because not all health professionals at each hospital participated in the focus groups. Despite this, the majority of respondents had worked in their current position for at least 4 years, so they could knowledgeably describe their stroke care service. The authors acknowledge the difficulty in capturing everything that is said during a focus group interview, especially when the interviews are not tape recorded. We did not have the resources available for this project to tape and to transcribe each of the focus group interviews. Nevertheless, we believe our processes for data collection ensured we captured the information in detail. This is because very few respondents made changes or additions to the data collected when they verified their transcript (and most changes were grammatical in nature rather than content-based). Respondents may also have been reluctant to answer questions about their hospital in front of their peers. However, participants were given the opportunity to individually verify the transcript and add further comments and then could submit this directly to the researchers securely via email.

Identifying both barriers and enablers to providing evidence-based stroke care is one of the first essential steps in implementing behavior change. Using time-limited facilitators was felt to be an important element in enhancing stroke care. The potential barriers identified in this study including the lack of a SU (at sites where this had not been established), inadequate availability to dedicated specialist clinical staff for stroke care, and limited executive support to achieve system change, need to be considered in the next phase of implementing changes in stroke care practices. Future research should include an assessment of the impact of the SNF role on patient care, as well as the changes in clinicians' perceptions about stroke care at the hospitals.

\section{Conclusion}

Our findings provide the first substantive qualitative evidence obtained from multiple hospitals about the common factors hospitals are trying to grapple with for improving stroke care, and whether SNFs might be an acceptable approach to overcoming many of the barriers. Understanding the factors that influence the provision of evidence-based stroke care in hospitals may explain why variations in care and patient outcomes exist and may be used to address barriers in future implementation studies.

\section{Acknowledgments}

This project was funded by the Department of Human Services, State Government of Victoria, as part of the Victorian Stroke Care Strategy on behalf of the Victorian Stroke Clinical Network. The authors thank the VSCN managers and SNFs for assistance with development and 
review of the interview schedule. We are grateful to the hospital staff and the SNFs who participated in the semistructured interviews for this qualitative analysis. We also acknowledge Ian Mosley for having contributed to data analysis for two hospitals and Hanna Burton for assistance with data compilation.

\section{Disclosure}

The authors report no conflicts of interest in this work.

\section{References}

1. Roger VL, Go AS, Lloyd-Jones DM, et al; American Heart Association Statistics Committee and Stroke Statistics Subcommittee. Heart disease and stroke statistics - 2012 update: a report from the American Heart Association. Circulation. 2012;125(1):e2-e220.

2. Senes S. How We Manage Stroke in Australia. Canberra: Australian Institute of Health and Welfare; 2006. Available from: http://www.aihw. gov.au/WorkArea/DownloadAsset.aspx?id=6442454967. Accessed March 11, 2014.

3. Cadilhac DA, Ibrahim J, Pearce DC, et al; SCOPES Study Group. Multicenter comparison of processes of care between Stroke Units and conventional care wards in Australia. Stroke. 2004;35(5):1035-1040.

4. Langhorne P, Pollock A; Stroke Unit Trialists' Collaboration. What are the components of effective stroke unit care? Age Ageing. 2002;31(5): $365-371$.

5. Stroke Unit Trialists' Collaboration. Organised inpatient (stroke unit) care for stroke. [Art. No.: CD000197], Cochrane Database of Systematic Reviews. 2013.

6. Zhu HF, Newcommon NN, Cooper ME, et al; Calgary Stroke Program. Impact of a stroke unit on length of hospital stay and in-hospital case fatality. Stroke. 2009;40(1):18-23.

7. Middleton S, McElduff P, Ward J, et al; QASC Trialists Group. Implementation of evidence-based treatment protocols to manage fever, hyperglycaemia, and swallowing dysfunction in acute stroke (QASC) a cluster randomised controlled trial. Lancet. 2011;378(9804):1699-1706.

8. Wardlaw JM, Murray V, Berge E, Del Zoppo GJ. Thrombolysis for acute ischaemic stroke. [Review]. Cochrane Database of Syst Rev. 2009;4:CD000213.

9. National Stroke Foundation. National Stroke Audit Acute Services. Clinical Audit Report 2013. Melbourne: National Stroke Foundation 2013. Available from: http://strokefoundation.com.au/site/media/ NSF687.ClinicalServicesAudit2013.Final_.pdf. Accessed March 11, 2014.

10. Cadilhac DA, Kilkenny MF, Longworth M, Pollack MR, Levi CR; Metropolitan Clinical Taskforce and Stroke Services New South Wales Coordinating Committee. Metropolitan-rural divide for stroke outcomes: do stroke units make a difference? Intern Med J. 2011;41(4):321-326.

11. Kwan J, Sandercock P. In-hospital care pathways for stroke. Cochrane Database of Systematic Reviews. 2004;(4): CD002924.

12. Victorian Government Department of Human Services. Stroke Care Strategy for Victoria. Springvale South, Victoria, Australia: Victorian Government Department of Human Services; 2007. Available from: http://docs.health.vic.gov.au/docs/doc/63C0F126DF44F8BDCA257 981000511B0/\$FILE/stroke_care_strategy.pdf. Accessed March 11, 2014.

13. Rycroft-Malone J. The PARIHS framework - a framework for guiding the implementation of evidence-based practice. J Nurs Care Qual. 2004;19(4):297-304.

14. Stetler CB, Legro MW, Rycroft-Malone J, et al. Role of “external facilitation" in implementation of research findings: a qualitative evaluation of facilitation experiences in the Veterans Health Administration. Implement Sci. 2006;1:23.
15. Cadilhac DA, Purvis T, Kilkenny MF, et al; New South Wales Strokes Services Coordinating Commitee; Agency for Clinical Innovation. Evaluation of rural stroke services: does implementation of coordinators and pathways improve care in rural hospitals? Stroke. 2013;44(10): 2848-2853.

16. Larsen LS, Neverett SG, Larsen RF. Clinical nurse specialist as facilitator of interdisciplinary collaborative program for adult sickle cell population. Clin Nurse Spec. 2001;15(1):15-22.

17. Mellor F, Foley T, Connolly M, Mercer V, Spanswick M. Role of a clinical facilitator in introducing an integrated care pathway for the care of the dying. Int J Palliat Nurs. 2004;10(10):497-501.

18. Donnellan C, Sweetman S, Shelley E. Implementing clinical guidelines in stroke: a qualitative study of perceived facilitators and barriers. Health Policy. 2013;111(3):234-244.

19. Stecksén A, Lundman B, Eriksson M, Glader EL, Asplund K. Implementing thrombolytic guidelines in stroke care: perceived facilitators and barriers. Qual Health Res. 2014;24(3):412-419.

20. Menon A, Bitensky NK, Straus S. Best practise use in stroke rehabilitation: from trials and tribulations to solutions! Disabil Rehabil. 2010;32(8):646-649.

21. McCluskey A, Vratsistas-Curto A, Schurr K. Barriers and enablers to implementing multiple stroke guideline recommendations: a qualitative study. BMC Health Serv Res. 2013;13:323.

22. McClusky A. Occupational therapists report a low level of knowlegde, skill and invovlement in evidence-based practice. Aust Occup Ther J. 2003;50(1):3-12.

23. Mays N, Pope C. Qualitative research in health care. Assessing quality in qualitative research. BMJ. 2000;320(7226):50-52.

24. Australian Institute of Health and Welfare. Rural, Regional and Remote Health: A Guide to Remoteness Classifications. Canberra: Australian Institute of Health and Welfare; 2004. Available from: http:/www.aihw. gov.au/WorkArea/DownloadAsset.aspx?id=6442459567. Accessed March 11, 2014

25. Glasgow RE, Davis CL, Funnell MM, Beck A. Implementing practical interventions to support chronic illness self-management. Jt Comm J Qual Saf. 2003;29(11):563-574.

26. National Stroke Foundation. Stroke Care Outcomes: Providing Effective Services (SCOPES)-An evaluation of Victorian Stroke Services: Final Report. National Stroke Foundation. 2002.

27. Flottorp S, Oxman A, Krause J, et al. A checklist for identifying determinants of practice: A systematic review and synthesis of frameworks and taxonomies of factors that prevent or enable improvments in healthcare professional practice. Implementation Science. 2013;8:35.

28. Patton M. Qualitative Evaluation and Research Methods. 2nd ed. Newbury Park, CA, USA: Sage Publications; 1990.

29. National Health and Medical Research Council. When does quality assurance in health care require independent ethical review? Advice to Institutions, Human Research Ethics Committees and Health Care Professionals. Canberra: National Health and Medical Research Council; 2003. Available from: http://www.nhmrc.gov.au/_files_nhmrc/ publications/attachments/e46.pdf. Accessed March 11, 2014.

30. Fuentes B, Díez-Tejedor E. Stroke units: many questions, some answers. Int J Stroke. 2009;4(1):28-37.

31. van Wijngaarden JD, Dirks M, Huijsman R, Niessen LW, Fabbricotti IN, Dippel DW; Promoting Acute Thrombolysis for Ischaemic Stroke (PRACTISE) Investigators. Hospital rates of thrombolysis for acute ischemic stroke: the influence of organizational culture. Stroke. 2009;40(10):3390-3392.

32. Burton CR, Fisher A, Green TL. The organisational context of nursing care in stroke units: a case study approach. Int J Nurs Stud. 2009;46(1):85-94.

33. Smith LN, Craig LE, Weir CJ, McAlpine CH. Stroke education for healthcare professionals: making it fit for purpose. Nurse Educ Today. 2008;28(3):337-347.

34. Alberts MJ, Hademenos G, Latchaw RE, et al. Recommendations for the establishment of primary stroke centers. Brain Attack Coalition. JAMA. 2000;283(23):3102-3109. 
35. Stroke Nurse Navigator Program [webpage on the Internet]. Elk Grove Village, IL, USA: Alexian Brothers Neurosciences Institute; 2014. [cited March 12, 2013]. Available from: http://www.alexianbrothershealth. org/neurosciences/services/stroke/stroke-nurse-navigator-program. Accessed March 12, 2013.

36. Nelson R. The clinical nurse leader. Am J Nurs. 2010;110(1):22-23.

37. National Stroke Foundation. Clinical Guidelines for Stroke Management 2010. Recommendations. Melbourne, Australia: National Stroke Foundation; 2010. Available from: http://strokefoundation.com.au/ site/media/Clinical_Guidelines_Acute_Management_Recommendations_2010.pdf. Accessed March 12, 2013.
38. Green T, Newcommon N. Advancing nursing practice: the role of the nurse practitioner in an acute stroke program. J Neurosci Nurs. 2006;38(Suppl 4):328-330.

39. Grimshaw J, Eccles M, Thomas R, et al. Toward evidence-based quality improvement. Evidence (and its limitations) of the effectiveness of guideline dissemination and implementation strategies 1966-1998. J Gen Intern Med. 2006;21 Suppl 2:S14-S20.

\section{Publish your work in this journal}

The Journal of Multidisciplinary Healthcare is an international, peerreviewed open-access journal that aims to represent and publish research in healthcare areas delivered by practitioners of different disciplines. This includes studies and reviews conducted by multidisciplinary teams as well as research which evaluates the results or conduct of such teams or healthcare processes in general. The journal covers a wide range of areas and welcomes submission from practitioners at all levels, from all over the world. The manuscript management system is completely online and includes a very quick and fair peer-review system. Visit http://www.dovepress.com/testimonials.php to read real quotes from published authors.

Submit your manuscript here: http://www.dovepress.com/journal-of-multidisciplinary-healthcare-journal 